\title{
Venous oxygen saturation is reduced and variable in central retinal vein occlusion
}

\author{
Umit Yolcu • Abdullah Ilhan • Fatih C. Gundogan • \\ Uzeyir Erdem
}

Received: 1 December 2014 / Accepted: 17 December 2014 /Published online: 10 January 2015

(C) Springer-Verlag Berlin Heidelberg 2015

Dear Editor,

We congratulate Eliasdottir et al. [1] for their study entitled 'Venous oxygen saturation is reduced and variable in central retinal vein occlusion'. The authors used a non-invasive spectrophotometric retinal oximeter for measuring hemoglobin oxygen saturation in retinal vessels. They concluded that central retinal vein occlusion (CRVO) eyes are hypoxic compared to fellow eyes, and hemoglobin oxygen saturation is increased between arterioles and venules. We would like to share our reservations about the study.

Fundus fluorescein angiography (FFA) and optical coherence tomography (OCT) are widely used for evaluation of CRVO cases. The authors also shared a patient's FFA and OCT results in figure legend 4 . Retinal oximetry is a recently developed diagnostic tool. We think that assessing the patients by OCT and FFA and comparing them with retinal oximetry results can provide precious clues for the pathogenesis and management of CRVO.
If available, we wonder if oximetric results of CRVO eyes with and without macular edema or CRVO eyes with or without retinal ischemia may be compared with each other. We think that its results also can be compared with FFA and OCT results in concordant and/or discordant sectors. We believe that these additional analyses will make it easy to reach reasonable inferences from the findings of the oximeter and increase the reliability of it.

Conflict of interest None of the authors have any conflict of interest with the submission.

\section{Reference}

1. Eliasdottir TS, Bragason D, Hardarson SH, Kristjansdottir G, Stefansson E (2014) Venous oxygen saturation is reduced and variable in central retinal vein occlusion. Graefes Arch Clin Exp Ophthalmol. doi:10.1007/s00417-014-2849-2

U. Yolcu $(\bowtie)$

Ophthalmology Service, Sarıkamıș Military Hospital, Kars 36500 ,

Turkey

e-mail: umit_yolcu@hotmail.com

\author{
A. Ilhan \\ Ophthalmology Service, ErzurumMilitary Hospital, Erzurum, \\ Turkey \\ F. C. Gundogan • U. Erdem \\ Department of Ophthalmology, GATA Medical School, Ankara, \\ Turkey
}

